\title{
Peptide conjugates of transportan 10 with antimicrobial and antifungal antibiotics
}

\author{
Katarzyna Olkiewicz ${ }^{1}$, Anna Łęgowska ${ }^{1}$, Natalia Ptaszyńska ${ }^{1}$, Agata Gitlin-Domagalska ${ }^{1}$, Dawid Dębowski ${ }^{1}$, Joanna \\ Okońska $^{1}$, Dorota Martynow ${ }^{2}$, Marcin Serocki $^{2}$, Sła womir Milewski ${ }^{2}$, Krzysztof Rolka $^{1}$ \\ ${ }^{1}$ Department of Molecular Biochemistry, Faculty of Chemistry, University of Gdańsk, Poland \\ ${ }^{2}$ Department of Pharmaceutical Technology and Biochemistry, Faculty of Chemistry, Gdańsk University of Technology, Poland \\ https://doi.org/10.17952/35EPS.2018.309
}

\section{Introduction}

Conjugates, in which two molecules differing by chemical and physical properties are connected, are a promising group of compounds. Conjugates consisting of a cell penetrating peptide (CPP) and a molecule with therapeutic properties are among most frequently studied compounds. CPPs can deliver to eukaryotic and prokaryotic cells a vast range of different biologically active compounds, such as proteins, nucleic acids, oligonucleotides, liposomes, nanoparticles, peptides, PNA, and low-molecular chemotherapeutic agents (e.g. antibiotics). Transportan(TP), reported by Langel group, is a chimeric peptide composed of the first 12 amino acid residues of neuropeptide galanin and 14 amino acid residues-long wasp venom peptide, mastoparan, connected via a lysine residue. A short variant, named TP10, with deletion of the N-terminal hexapeptide, retains the efficient cell penetration property of the parent compound with significantly less potential side effects [1].

In this paper we report synthesis and biological studies of peptide conjugates composed of TP10 and antibiotics: levofloxacin, ciprofloxacin and fluconazole. Three different linkers (methylene carbonyl, amide and disulfide bridge) were used to connect both components. TP10 may not only help to transport the antibiotic across the microbial cell membranes, but having intrinsic antimicrobial activity, it can potentiate or even change the molecular mechanism of action of the conjugated antibiotic. In order to determine the cellular uptake of TP10 and its conjugate with ciprofloxacin, their fluorescently-labeled analogues were also synthesized (TP10(F)- $\mathrm{NH}_{2}$ and CIP-TP10(F)-NH $\mathrm{NH}_{2}$. The chemical formulas of antibiotics and synthesized compounds are shown in Fig. 1.
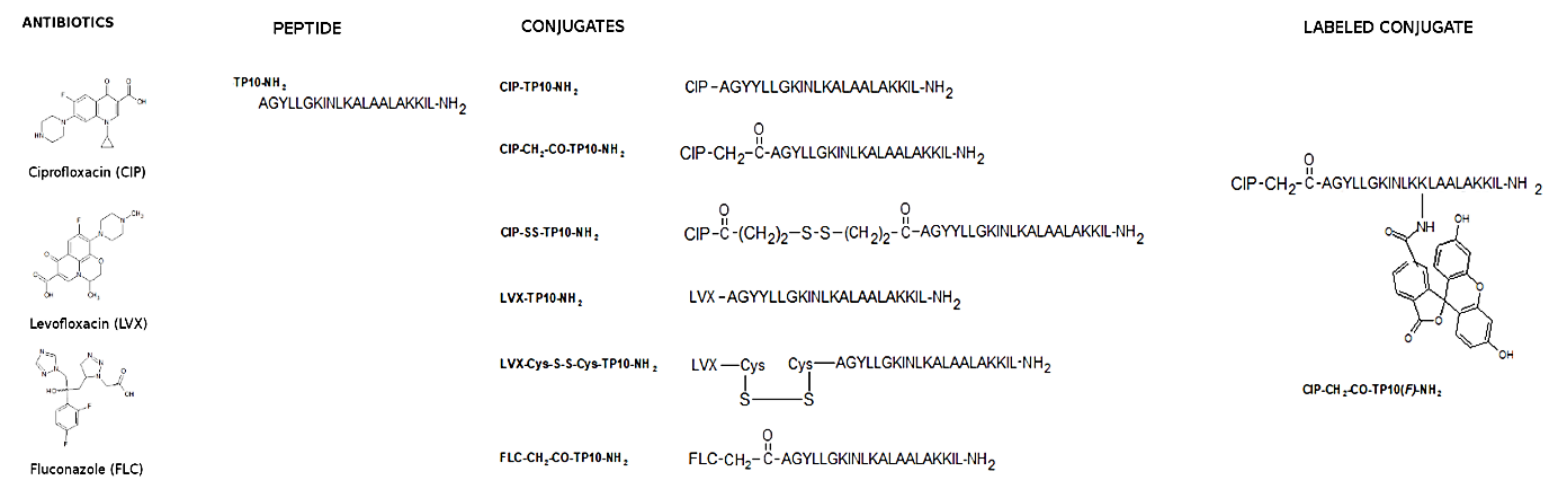

Figure 1: Chemical structures of antibiotics, TP10 and peptide conjugates.

\section{Results and Discussion}

All conjugates were obtained using solid phase synthesis and standard 9-fluorenylmethoxycarbonyl (Fmoc) chemistry. In CIP-TP10- $\mathrm{NH}_{2}$ and LVX-TP10- $\mathrm{NH}_{2}$ amide bond was formed between carboxyl group of CIP or LVX and peptide $\alpha$-amino group. In CIP-CH2-CO-TP10- $\mathrm{NH}_{2}$, methylene carbonyl linker was applied to connect antibiotic through its amine to the N-terminus of TP10. Intermolecular disulfide bridge, spanning both components in CIP-SS-TP10- $\mathrm{NH}_{2}$ and LVX-Cys-S-S-Cys-TP10- $\mathrm{NH}_{2}$, was formed using Lomant's reagent - dithio-bis(succinimidyl propionate) or between two Cys residues.

FLC-CH2-CO-TP10- $\mathrm{NH}_{2}$ was synthesized via modified Huisgen 1,3-dipolar cycloaddition, known also as click reaction. This required synthesis of the FLC precursor (1 in Fig. 2.) containing alkyne group, which was obtained by the method described by Pore et al. [2], and then a click reaction of 2-(2,4-difluorophenyl)-1(1H-1,2,4-triazol-1-yl)pent-4-yn-2-ol with a peptide containing azidoacetyl moiety attached to $\alpha$-amino group was 
performed. In order to determine the cellular uptake, fluorescently-labeled CIP-CH2-CO-TP10(F)-NH $\mathrm{NH}_{2}$ and TP10(F)- $\mathrm{NH}_{2}$ were also synthesized. In both compounds, 5(6)-carboxyfluorescein was attached to the $\varepsilon$-amino group of peptide's Lys that replaced Leu in position 12.<smiles>C#CC[C@](O)(Cn1cncn1)c1ccc(F)cc1F</smiles>

Figure 2: Synthesis of FLC-CH2-CO-TP10- $\mathrm{NH}_{2}$.

All conjugates and their parent compoundswere investigated for fungicidal activity against four fungal strains: $C$. albicans ATCC 10231, C. albicans SC 5314, C. krusei DSM 6128, and C. glabrata DSM 11226, and antibacterial activity against five bacterial strains, three Gram-positive: S. aureus ATCC 25923, S. aureus ATCC 29213, S. epidermidis ATCC 12228, and two Gram-negative (E.coli ATCC 25922, P. aeruginosaATCC 27853). TP10- $\mathrm{NH}_{2}$, levofloxacin and ciprofloxacin did not exhibit antifungal activity at concentrations up to $100 \mu \mathrm{g} / \mathrm{ml}$; however, two conjugates with ciprofloxacin CIP-TP10- $\mathrm{NH}_{2}$ and CIP-SS-TP10- $\mathrm{NH}_{2}$ were active against both $C$. albicans strains $\left(\mathrm{MIC}_{50}\right.$ values determined for CIP-TP10- $\mathrm{NH}_{2}$ were 62 and $30 \mu \mathrm{g} / \mathrm{mL}$, respectively and for CIPSS-TP10- $\mathrm{NH}_{2} \mathrm{MIC}_{50}$ were 24 and $34 \mu \mathrm{g} / \mathrm{mL}$, respectively). Unfortunately, FLC-CH2-CO-TP10- $\mathrm{NH}_{2}$ was not active towards tested fungal strains. TP10 did not display antibacterial activity at concentrations up to $200 \mu \mathrm{g} / \mathrm{ml}$, whereas its two conjugates with ciprofloxacin CIP-TP10- $\mathrm{NH}_{2}$ and CIP-SS-TP10- $\mathrm{NH}_{2}$ and one with levofloxacin LVX-Cys-S-S-Cys-TP10-NH 2 inhibited growth of all studied bacteria (Table 1). Interestingly, fluconazole conjugate was also found active towards bacteria. To assess the cytotoxic effect of TP10, ciprofloxacin and its two conjugates, we estimated $\mathrm{IC}_{50}$ values (using MTT assay) for two human cell lines: liver cancer cells (HepG2) and cell line of embryonic kidney (HEK 293), as well as a cell line isolated from kidney of 3-4 weeks old male pig (LLC-PK1). The conjugates were slightly more cytotoxic than their constituents; however, they induced a relatively low cytotoxic effect in comparison with antibiotics widely used in clinic. Microscopic observations showed that TP10(F)- $\mathrm{NH}_{2}$ as well as its conjugate CIP-CH2-COTP10(F)- $\mathrm{NH}_{2}$ penetrate well into C. albicans SC 5314, and C. albicans ATCC 10231.

Table 1: MIC values determined for selected bacterial strains

\begin{tabular}{|c|c|c|c|c|c|c|c|c|c|c|}
\hline \multirow{4}{*}{ Compound } & \multicolumn{10}{|c|}{$\operatorname{MIC}[\mu \mathrm{g} / \mathrm{ml}]$} \\
\hline & \multicolumn{6}{|c|}{ Gram $(+)$} & \multicolumn{4}{|c|}{ Gram (-) } \\
\hline & \multicolumn{2}{|c|}{$\begin{array}{c}\text { S. aureus } \\
\text { ATCC } 25923\end{array}$} & \multicolumn{2}{|c|}{$\begin{array}{c}\text { S. aureus } \\
\text { ATCC } 29213\end{array}$} & \multicolumn{2}{|c|}{$\begin{array}{c}\text { S. epidermidis } \\
\text { ATCC } 12228\end{array}$} & \multicolumn{2}{|c|}{$\begin{array}{c}\text { E. coli } \\
\text { ATCC } 25922\end{array}$} & \multicolumn{2}{|c|}{$\begin{array}{l}\text { P. aeruginosa } \\
\text { ATCC } 27853\end{array}$} \\
\hline & $\mathrm{MIC}_{90}$ & $\mathrm{MIC}_{50}$ & $\mathrm{MIC}_{90}$ & $\mathrm{MIC}_{50}$ & $\mathrm{MIC}_{90}$ & $\mathrm{MIC}_{50}$ & $\mathrm{MIC}_{90}$ & $\mathrm{MIC}_{50}$ & $\mathrm{MIC}_{90}$ & $\mathrm{MIC}_{50}$ \\
\hline Ciprofloxacin (CIP) & $<0.3$ & $<0.3$ & $<0.3$ & $<0.3$ & 0.9 & 0.2 & $<0.3$ & $<0.3$ & $<0.3$ & $<0.3$ \\
\hline Levofloxacin (LVX) & $<0.3$ & $<0.3$ & $<0.3$ & $<0.3$ & 2.2 & 1.5 & $<0.3$ & $<0.3$ & 1.5 & 1.1 \\
\hline Fluconazole (FLC) & $>250$ & $>250$ & $>250$ & $>250$ & $>250$ & $>250$ & $>250$ & $>250$ & $>250$ & $>250$ \\
\hline TP10 & $>300$ & 257 & $>300$ & 231 & $>300$ & 215 & 246 & 121 & $>300$ & 284 \\
\hline CIP-CH${ }_{2} \mathrm{CO}-\mathrm{TP} 10$ & 295 & 132 & 290 & 198 & 215 & 102 & 15 & 9 & 256 & 115 \\
\hline CIP-TP10 & 4 & 4 & 2 & 2 & 4 & 4 & 4 & 4 & 8 & 8 \\
\hline CIP-SS-TP10 & 1.1 & 0.7 & 1.3 & 0.8 & 1.6 & 0.2 & $<0.3$ & $<0.3$ & 1.1 & 0.8 \\
\hline LVX-TP10 & $>>300$ & 199 & $>>300$ & 289 & 274 & 211 & 74 & 28 & 164 & 140 \\
\hline LVX-Cys-SS-Cys-TP10 & 62 & 62 & 16 & 16 & 8 & 8 & 8 & 8 & 62 & 31 \\
\hline FLC-TP10 & 16 & 16 & 8 & 8 & 8 & 8 & 8 & 8 & 62 & 62 \\
\hline
\end{tabular}

In conclusion, we obtained six new conjugates composed of antibiotic (ciprofloxacin, levofloxacin or fluconazole) and TP10- $\mathrm{NH}_{2}$. Two ciprofloxacin conjugates CIP-TP10- $\mathrm{NH}_{2}$ and CIP-SS-TP10- $\mathrm{NH}_{2}$ exhibited fungicidal activity against $C$. albicans 10231 and $C$. albicans SC 5314, even though their constituents were inactive. These conjugates displayed also antibacterial activity, whereas $\mathrm{TP} 10-\mathrm{NH}_{2}$ did not inhibit bacterial growth. It is worth noting that the activity of conjugate CIP-SS-TP10- $\mathrm{NH}_{2}$ was the same as of ciprofloxacin. 


\section{Acknowledgements}

This work was supported by the National Science Centre (NCN) under grant No UMO-2016/21/B/ST5/00101.

\section{References}

[1] M. Pooga, M. Hallbrink, M. Zorko, Ü. Langel, FASEB J., 1998, 12, 67-77;

[2] Pore V.S., Aher N.G., Kumarb M., Shukla P. K., Tetrahedron,2006, 62, 11178-11186; doi. 